\title{
Perspectivas del ecoturismo, fundamentos legales y desarrollo sostenible
} Perspectives of ecotourism, legal foundations and sustainable development

* Leo Ruperti León, ** Argenis Montilla Pacheco, *** Leydy Cevallos Barberán

*leo.ruperti@uleam.edu.ec, **argenismontilla@hotmail.com, ***leydy.cevallosb@gmial.com

Fecha de recepción: 08/10/2018 Fecha de aceptación: 17/02/2019 Publicado: $30 / 06 / 2019$

\section{Resumen}

El presente trabajo tiene como objetivo discutir aspectos que guardan relación con las perspectivas del turismo ecológico y el desarrollo sostenible. Se trata de un ensayo en el que, a partir del análisis de material bibliográfico los autores exponen su punto de vista sobre el tema en cuestión. De igual manera se aborda la parte jurídica, pues actualmente se ponen en práctica instrumentos legales novedosos que regulan el manejo del ambiente, de los recursos naturales y del turismo ecológico, muchos de ellos de alcance local, nacional y mundial. El ecoturismo como modalidad resulta ser una nueva y predominante tendencia del turismo, en la cual, el turista busca no solo calidad en los servicios, sino el contacto directo con la naturaleza, especialmente con aquellos espacios que por su alto valor ecológico se han constituido con carácter de oficialidad en áreas protegidas, regidas por leyes que sobre la materia se han formulado en distintas regiones del mundo. De esa forma, el ecoturismo como actividad económica debe sustentarse en los principios del desarrollo sostenible, que es al mismo tiempo una herramienta para mejorar las relaciones de los seres humanos con su entorno, y en consecuencia, para hacer uso racional y consciente de los recursos y atractivos turísticos que oferta la naturaleza.

Palabras clave: Ecoturismo, ecosistema, medio ambiente natural, desarrollo sostenible, fundamentos legales del ecoturismo. 


\begin{abstract}
The objective of this work is to discuss aspects that are related to the perspectives of ecological tourism and sustainable development. This is an essay in which, from the analysis of bibliographic material, the authors present their point of view on the subject in question. Likewise, the legal part is addressed, since currently innovative legal instruments are being implemented that regulate the management of the environment, natural resources and ecological tourism, many of them of local, national and global scope. Ecotourism as a modality turns out to be a new and predominant tendency of tourism, in which tourists seek not only quality services, but also direct contact with nature, especially those spaces that due to their high ecological value have been constituted with character of officiality in protected areas, governed by laws that on the matter have been formulated in different regions of the world. In this way, ecotourism as an economic activity must be based on the principles of sustainable development, which is at the same time a tool to improve the relationships of human beings with their environment, and consequently, to make rational and conscious use of resources and tourist attractions offered by nature.
\end{abstract}

Keywords: Ecotourism, ecosystem, natural environment, sustainable development, legal foundations of ecotourism.

\title{
Introducción
}

Posterior a la Segunda Guerra Mundial se suscitaron cambios significativos en la sociedad, estos afectaron sensiblemente el estilo de vida de muchos grupos humanos, entre ellos lo que tiene que ver con la recreación y el descanso. Fue así que el turismo de masas se perfiló a escala planetaria como una de las modalidades más importantes (Bringas \& Ojeda, 2000), y con claras perspectivas de crecimiento señaladas por la Organización Mundial del Turismo a finales del siglo XX (OMT, 1998). 
La movilidad ha estado presente en toda la historia de la humanidad. No es novedad. Lo que sí es nuevo es el impacto de esas movilizaciones en el siglo XX. Esto innegablemente genera un creciente interés por su estudio y, en consecuencia, una reflexión científica que conlleva a una conciencia ambiental con la divulgación masiva de investigaciones de la degradación del ambiente a nivel global. El turismo de masas es altamente cuestionado, pues son ciertas las evidencias de los daños que esta actividad ha causado sobre los ecosistemas en distintas regiones de la Tierra. De manera que surgen nuevas modalidades del turismo, con un enfoque distinto que intenta poner por delante el raciocinio, el respeto por la naturaleza y la cultura ancestral de los pueblos.

Dentro de la modalidad de turismo alternativo al turismo masivo se encuentra el turismo ecológico que parte del principio de sostenibilidad, consustanciado con el mantenimiento de la armonía hombre - naturaleza. Como define Ibáñez \& Reyna (2011), es un nuevo movimiento conservatorio basado en la industria turística, que se define como viajes responsables que conservan el entorno y sostienen el bienestar de la comunidad local.

El concepto Ecoturismo tiene su origen en Costa Rica en el año 1985 cuando un conjunto de biólogos y naturistas encabezados por el Dr. Rolando Mendoza lo utilizan por primera vez en investigaciones para la preservación del medio ambiente. De allí, quien guía un grupo de personas cuando hacen ecoturismo, pasó a denominársele naturalista, debido a su interés en propiciar el conocimiento de la naturaleza en los visitantes. Ello se convirtió en un elemento diferenciador a lo que hasta ese momento se conocía como guía turístico.

A partir de ese momento dentro del turismo comienzan a desarrollarse eventos globales que van transformando las categorías de análisis de esta disciplina. En la medida que se profundiza en su estudio los pasos iniciales del ecoturismo avanzan hacia una nueva concepción que permiten a la Organización Mundial del Turismo concluir que sin importar el área de especialidad y el nombre que se le asigne, todo turismo debe ser sostenible. 
Desde entonces el ecoturismo se yuxtapone al turismo naturalista y tangencialmente se asocia al turismo de aventura y rural, teniendo todos como centro el turismo sostenible.

Hoy día cobra gran interés el ecoturismo en el concierto de las actividades económicas, fundamentalmente de aquellas que comprende el sector servicios. Por tanto, se percibe como una fórmula para generar ingresos económicos sobre la base del aprovechamiento de las potencialidades que ofrecen las áreas naturales protegidas. En este caso, todas estas actividades enmarcadas en los postulados del desarrollo sostenible, concepto que emerge a raíz del deterioro generalizado de la naturaleza, la afectación de la salud de la población, la escasez de alimentos, agua, materia prima, y un aumento en la incidencia de desastres naturales (Alaña et al., 2017).

En lo que respecta a la parte legal orientada a proteger la naturaleza y regular el turismo, debe señalarse que hasta la fecha son numerosos los instrumentos jurídicos elaborados con carácter local, regional, nacional y mundial; no obstante, los alcances esperados de su aplicación no han sido lo suficientemente relevantes como para afirmar que sean la clave para resguardar el ambiente y los recursos o atractivos turísticos. Es así como las disposiciones legales que rigen la materia no han podido evitar que el daño ambiental se haga presente en áreas naturales protegidas y en ecosistemas de altos niveles de fragilidad, y mucho menos en otros espacios que carecen de amparo en materia jurídica.

En ese mismo orden de ideas, dentro del marco del turismo, de acuerdo a Maldonado, (2006), las políticas aplicadas durante las últimas décadas para fomentar el desarrollo social, disminuir la pobreza y frenar la degradación del ambiente, no han logrado concretar los resultados esperados. Así mismo, agrega el autor, que las causas que han frustrado esas esperanzas en el ámbito mundial son bien conocidas, entre ellas la falta de voluntad política y fuertes presiones del modelo de producción y consumo dominante; todo lo cual se ha conjugado con la no aplicación al pie de la letra de la reglamentación jurídica pertinente. 
Finalmente, en este trabajo, se quiere llamar a reflexión respecto a la importancia que tiene la práctica de un turismo consciente, llevado a cabo sobre la base de los principios de la sostenibilidad, y desde luego, del respeto a los instrumentos legales que sobre el particular se han instituido en distintos países. Si se hace de esta forma, es posible que los recursos naturales que se aprovechan para el turismo ecológico se puedan renovar en la misma medida en la que van siendo utilizados, con lo cual, estos podrán seguir dando sustento a las generaciones presentes, al tiempo que se garantizaría para las generaciones del futuro.

\section{Conceptualización del ecoturismo, estado actual y perspectivas}

El término ecoturismo se ha utilizado de diferentes maneras. Para la promoción de múltiples actividades turísticas efectuadas de manera directa sobre espacios naturales, en donde los turistas demuestran interés y se sienten atraídos por conocer desde muy cerca la naturaleza y la riqueza cultura de la población anfitriona (Fennell, 2001; Vanegas 2006). Visto de esa manera, el ecoturismo se identifica como turismo naturalista, compuesto por una gama amplia de destinos y ofertas de atractivos naturales diversos, entre ellos, ríos, cascadas, volcanes y otros.

El ecoturismo, también es definido como una modalidad turística cuyo objetivo es el aprovechamiento, uso y conservación de los recursos naturales y culturales desde las comunidades locales (Bassotti, 2003). Aunque este tipo de turismo, en teoría no debería generar daños ambientales en los destinos geográficos, en la práctica sí lo hace (Agüera, 2014a), especialmente cuando no se toman medidas para evitarlo. De acuerdo a esta definición, el ecoturismo se confunde con aprovechamiento y uso de recursos desde lo local dejando aspectos tan importantes como la contemplación responsable con fines educativos sin considerar. 
La anterior afirmación se sustenta en los planteamientos de Maldonado, (2006), quien describe que el turismo no es una actividad inocua, pues produce cambios en las sociedades donde se implanta; y es, además, una acción ambivalente, por cuanto implica impactos positivos y adversos, de los cuales algunos resultan irreversibles, fundamentalmente cuando se trata de sistemas ecológicos de alta fragilidad. En ese mismo contexto, ya desde hace casi tres décadas, Achana et al., (1994) comentaban que el ecoturismo es un campo en franca evolución y que experimentará una variedad de transformaciones dolorosas al igual que la experimentaron otras actividades, entre ellas la agricultura y la industria.

En cuanto al estado actual del turismo, hay que precisar primero que la disciplina científica que se enfoca a su estudio desde una perspectiva del desarrollo sustentable. El turismo ecológico, como también se denomina, toma en cuenta los ecosistemas y los recursos y atractivos turísticos naturales como unidades fundamentales de análisis; por lo tanto, este tipo de investigación se viene ocupando de buscar información, acceder al conocimiento de todo cuanto tenga que ver con el patrimonio natural y ponerlo a disposición de quien lo demande.

Entre el turismo y las áreas protegidas ha existido un vínculo indisoluble (Eagles et al., 2002). Se ha debatido acerca de tratar las áreas protegidas con una visión científica y excluyente de cualquier actividad humana o, por el contrario, propiciar categorías de manejo de estos espacios con restricciones. La evolución de este pensamiento lleva al impulso de programas globales de conservación de áreas protegidas que ha dado como resultado una modalidad muy particular dentro de la disciplina turística conocida como ecoturismo. Esta modalidad representa hoy día una importante fuente de ingresos económicos en distintos países, y de manera especial en aquellos que por sus condiciones geográficas son el asiento de muchos atractivos.

Las áreas protegidas se constituyen como ambientes portadores de una enorme expresión de vida silvestre, tanto en flora como en fauna (Corraliza et al., 2002), razón por la cual atraen 
numerosas cantidades de turistas, en la mayoría de las veces procedentes de grandes centros urbanos (Flores, 2008), donde el stress típico de las ciudades hace que los individuos sientan la necesidad de experiencias de vida sana, en áreas naturales, donde la tranquilidad del ambiente y la belleza de los paisajes se articulen para ofrecer espacios de descanso y recreación.

Entre los beneficios que actualmente produce el ecoturismo destacan los económicos, pues según Agüera, (2014b), impulsa la creación de empleos y de nuevas empresas generadoras de ingresos económicos en su respectivo destino; además, aporta en beneficios sociales, porque contribuye, tal como apunta el autor, a mejorar de forma integral la calidad de vida de las familias que conforman las comunidades locales receptoras del turista.

En el mismo orden de ideas, el ecoturismo aporta sumas significativas de divisas en muchos naciones, así lo corroboran las cifras, pues ya desde la última década del pasado siglo XX, la Organización Mundial del Turismo (OMT, 2007) afirmaba que el turismo a nivel mundial tenía un crecimiento anual del $4 \%$, mientras que el turismo de naturaleza, o ecoturismo, crecía anualmente entre el 10\% y el 25\% aproximadamente. Por ejemplo, en Colombia, donde esta tipología de turismo vive actualmente un auge, el sistema de Parque Nacionales, solo por concepto de taquilla alcanzó una recaudación de 2.500 millones de pesos entre los años 2000 y 2003 (Fonseca et al., 2013).

La relevancia y, de manera especial, el valor estético y escénico de determinados espacios geográficos, potencialmente los convierte en atributos claves para su consolidación como destino ecoturístico. Partiendo de allí, las perspectivas de desarrollo y crecimiento de esta modalidad del turismo son realmente prometedoras, sobre todo en aquellos lugares donde las condiciones geoambientales se enlazan para mostrar paisajes de particular belleza, como, glaciares, montañas, ríos, lagos, cuevas, bosques, páramos, médanos, esteros y otros. Con todo ello, el ecoturismo contribuirá a que los visitantes de un destino puedan conocer y 
disfrutar del patrimonio natural, y de manera simultánea se estarían fomentando actitudes en favor de la conservación de los sistemas ecológicos y los recursos naturales inmersos en ellos.

\section{Fundamentos legales que rigen el ecoturismo a distintas escalas}

Toda actividad que involucre acciones sobre espacios naturales, entre ellas las que derivan del ecoturismo se rige por disposiciones legales de diferente alcance, es decir, mundial, nacional, regional y local. A nivel internacional, en el marco de distintos acuerdos y convenios se han formulado disposiciones para proteger la naturaleza. Uno de ellos es el Convenio sobre la Diversidad Biológica (CDB), dado en Nairobi, capital de la República de Kenia en el año 1992.

El Convenio sobre Diversidad Biológica (CDB) tiene como objetivo primordial la conservación de la diversidad biológica, el uso sostenible y racional de sus componentes y la participación igualitaria de la población en los beneficios que resultan del aprovechamiento de los recursos genéticos (Van Dam, 2001; Bravo, 2002). De igual forma, este convenio se considera como el primer acuerdo mundial que aborda integralmente los componentes de los ecosistemas al tiempo que promueve con sus disposiciones la cooperación científica y tecnológica entre los países miembros. Este convenio, así como muchos otros, favorece el desarrollo del ecoturismo, pues protege los recursos y atractivos que impulsan esta actividad económica.

La Convención sobre la Conservación de las Especies Migratorias de Animales Silvestres, conocida como el Convenio de Bonn, celebrado en 1979 en Bonn, Alemania, es otro de los tratados importantes que persigue salvaguardar la fauna en ecosistemas ubicados en más de un centenar de países signatarios de la tal convención. Desde esta convención se hacen esfuerzos mancomunados orientados a la protección estricta de la biodiversidad faunística a partir de la creación de obligaciones que deben cumplir todos los Estados firmantes. 
Para evitar, regular y controlar la comercialización de plantas y animales se creó en Washington DC, en el año 1973 la Convención sobre el Comercio Internacional de Especies Amenazadas de Fauna y Flora Silvestres. Es un acuerdo de rango internacional que además tiene como finalidad garantizar que las actividades comerciales de especies de flora y fauna no comprometa su supervivencia en su hábitat (Dialhy \& Herrera, 2011). A la fecha, los resultados de este convenio son satisfactorios, pues ninguna de las 30.000 especies se ha extinguido.

Para brindar protección a los cuerpos de agua de alto valor ecológico se creó la Convención Relativa a los Humedales de Importancia Internacional (RAMSAR). Esta convención tuvo sus orígenes en la ciudad de Ramsar, Irán, específicamente en el año 1971, entrando en vigencia 4 años más tarde, es decir en 1975. Progresivamente muchos países se han sumado a esta convención, de tal forma que a lo largo y ancho de la geografía mundial, se suman cada vez más humedales a la categoría de sitio Ramsar (Molina, 2012).

Los resultados de esta convención resultan ser muy valiosos, pues a la fecha la lista de humedales en esta categoría alcanza más de 1900 sitios Ramsar, cuya cobertura total es equivalente a una superficie de $1.900 .000 \mathrm{~km}^{2}$ o 190.000 .000 de hectáreas. De esta manera, el ecoturismo se ve fortalecido, pues los humedales son espacios de gran interés para quienes sienten atracción por determinadas prácticas que se realizan dentro de esta modalidad, siendo una de ellas el aviturismo.

A nivel nacional, cada Estado dispone de normas que pretenden regular las acciones que involucran intervención del paisaje, el aprovechamiento de los recursos naturales y el ecoturismo. La Constitución de cada país, al menos de un importante número de ellos dispone de muchos artículos que rigen la materia. A manera de ejemplo puede citarse el artículo 2 de la Constitución Nacional del Ecuador (2008), el cual textualmente indica que: "Las políticas de gestión ambiental se aplicarán de manera transversal y serán de obligatorio cumplimiento 
por parte del Estado en todos sus niveles y por todas las personas naturales o jurídicas en el territorio nacional.”

En el mismo orden de ideas, la Constitución de la República de Colombia (1991), en el Capítulo 3, de Los Derechos Colectivos y del Ambiente, específicamente en el artículo 79, señala que "Todas las personas tienen derecho a gozar de un ambiente sano. La ley garantizará la participación de la comunidad en las decisiones que puedan afectarlo. Es deber del Estado proteger la diversidad e integridad del ambiente, conservar las áreas de especial importancia ecológica y fomentar la educación para el logro de estos fines”.

Por su parte, Venezuela dispone igualmente de diversos instrumentos jurídicos, que regulan el ámbito ambiental y el turístico. En tal sentido, la Constitución Nacional (1999), establece en el Capítulo IX, de los Derechos Ambientales, artículo 128, que "El Estado protegerá el ambiente, la diversidad biológica, los recursos genéticos, los procesos ecológicos los parques nacionales y monumentos naturales y demás áreas de especial importancia ecológica".

Otros no menos importantes instrumentos jurídicos de alcance nacional lo constituyen numerosas leyes creadas en cada país, así como ordenanzas de alcance estrictamente local, cuyo ámbito de aplicación se circunscribe a la geografía de cada cantón o municipio, según sea la división política territorial de cada país.

A manera de síntesis, puede señalarse que la norma jurídica, aun cuando representa avances significativos en cuanto a protección ambiental, en ninguno de los contextos espaciales descritos ha sido suficiente para frenar los procesos degradantes del ambiente, ni siquiera aquellos que se realizan en el marco de las actividades de ecoturismo; por tanto, todavía se está en deuda respecto a suprimir las preocupaciones planteadas por Rachell Carson en su libro "Primavera Silenciosa", y más aun de alcanzar la ansiada armonía con la 
madre naturaleza, promulgada en el siglo XII por San Francisco de Asís en su magistral obra "El Cántico de las Criaturas".

\section{Desarrollo sostenible}

El término desarrollo sostenible es de uso relativamente nuevo, y según Escobar (1995), aparece en condiciones históricas muy específicas, vinculadas con la problematización global de la relación hombre naturaleza, debido al incremento de la degradación del ambiente y de los ecosistemas. Artaraz, (2002) manifiesta que este concepto aparece por primera vez de forma oficial en 1987 en el Informe Brundtland referido al futuro del planeta y la relación ambiente - desarrollo; y que además se entiende como aquello que satisface las necesidades presentes asegurando al mismo tiempo la posibilidad de que las generaciones futuras puedan satisfacer sus propias necesidades.

Respecto al mismo término, La Unión Mundial para la Naturaleza (1993) define desarrollo sostenible como un proceso que permite el desarrollo sin degradar y agotar los recursos que lo hacen posible. Añade que tal objetivo se alcanza con una gestión eficiente y racional de los recursos, dando oportunidad a su renovación al mismo ritmo en que se van utilizando, pues es la estrategia acertada para que puedan dar sustento a las nuevas generaciones.

Desde esa óptica, el concepto de sostenibilidad es aplicado a la industria del turismo, y en específico al ecoturismo, lo cual constituye un reto complejo, de largo aliento que abriga esperanzas para el presente milenio (Maldonado, 2006). El reto, agrega el autor, es tanto más complejo en cuanto que el turismo es una de las actividades más relevantes a escala global, pues ha registrado un sostenido ritmo de crecimiento con implicaciones favorables en muchas ramas de la actividad económica (Maldonado, 2006), entre ellas transporte, gastronomía y recreación. 
Vanegas, (2006), asegura que su crecimiento, especialmente a partir de los años 80 del pasado siglo XX, está en función de los beneficios económicos que provee como turismo en la naturaleza. Añade el autor que el incentivo económico que genera el ecoturismo más allá de sus principios, ha dado origen a la expansión de este tipo de actividad en diferentes regiones del mundo, pero con mayor fuerza en las áreas protegidas, en las cuales, aun cuando disponen de normas que intentan regular su uso se evidencian impactos negativos.

El ecoturismo al alcanzar importantes niveles de expansión y generar alta rentabilidad ha ignorado sus efectos negativos, socioculturales y ambientales; igualmente al convertirse en turismo de masas muestra su carácter insostenible, pues deja de ser totalmente cierto su fundamento ecológico y con ello el mantenimiento de equilibrio en los ecosistemas donde se practica (Vanegas, 2006). Sobre el mismo tema Gurría, (1996), señalaba que los costos ecológicos y sociales se pagan a cambio de los beneficios económicos y cuando aquellos no son analizados, evaluados y controlados, los costos pueden superar los beneficios con resultados muchas veces lamentables ya evidentes en diversas regiones de la geografía mundial.

Por lo anterior, se hace impostergable formular nuevos mecanismos en el marco del desarrollo sostenible, que conduzcan a la plena armonía hombre naturaleza, pero además para hacer del ecoturismo una actividad francamente sostenible, tomando en cuenta que puede dar resultados altamente favorables, entre ellos lograr la Integración a las actividades turísticas de los pueblos anfitriones, comprender la repercusión de los efectos adversos de esta modalidad turística sobre los sistemas ecológicos, promover la creación de establecimientos de servicios turísticos y contribuir con más plazas de trabajo. 


\section{Conclusiones}

El ecoturismo es una actividad de singular importancia dentro del conjunto de actividades económicas, especialmente porque se basa en el disfrute, contemplación y aprovechamiento racional y consciente de los recursos naturales en beneficio de las distintas comunidades locales. Asimismo, se precisa divulgar y diseñar continuamente estrategias para que su promoción respete los ecosistemas.

Debido a la creciente demanda de conocimiento de la naturaleza en sus estados más prístinos, mayor conciencia ambiental y la necesidad de espacios propicios para el descanso y la recreación, el ecoturismo se perfila como una modalidad de enormes perspectivas a escala mundial, sobre todo en aquellos espacios que por sus condiciones naturales sintetizan todos las características del ecoturismo como modalidad: turismo responsable, disfrutar sin perturbar y apreciar, estudiar atractivos naturales y culturales desde una perspectiva sustentable.

Desde el punto de vista de las bases legales, ecoturismo cuenta con el amparo de diversos instrumentos jurídicos, a nivel internacional, nacional, regional y local; sin embargo, es preciso avanzar aún más en ese sentido, y crear condiciones que faciliten su cumplimiento, con lo cual, esta actividad económica sería sostenible en el sentido más estricto de la palabra.

Sensibilizar a la colectividad acerca de la importancia de conservar la naturaleza, seguramente provocará conductas tendientes a su protección. En ese sentido, el ecoturismo se vería ampliamente beneficiado, y tendría la posibilidad de contribuir en la economía y la cultura de las comunidades o espacios anfitriones. 


\section{Referencias bibliográficas}

Achana, F., Haysmith, L., \& Harvey, J. (1994). Definición de ecoturismo. Recuperado de: http://www.sidalc.net/cgi-

$\underline{\text { bin/wxis.exe/?IsisScript=GREYLIT } \text {.xis\&method=post } \& \text { formato=2\&cantidad }=1 \& \text { expresio }}$ $\underline{\mathrm{n}=\mathrm{mfn}=016574 .}$.

Agüera, F. (2014a). Los impactos económicos, sociales y medioambientales negativos en el ecoturismo: una revisión de la literatura/economic, social and environmental negative impacts negative in ecotourism: a review of the literature. Nómadas, 42(2), 1. Recuperado de: http://revistas.ucm.es/index.php/NOMA/article/viewFile/48781/45528

Agüera, F. (2014b). Turismo y cooperación al desarrollo: Un análisis de los beneficios del ecoturismo para los destinos. El Periplo Sustentable: revista de turismo, desarrollo y competitividad, (26), 47-66.

Alaña Castillo, T. P., Capa Benítez, L. B., \& Sotomayor Pereira, J. G. (2017). Desarrollo sostenible y evolución de la legislación ambiental en las MIPYMES del Ecuador. Universidad y Sociedad [seriada en línea], 9 (1), pp. 91-99. Recuperado de http://rus.ucf.edu.cu/

Artaraz, M. (2002). Teoría de las tres dimensiones de desarrollo sostenible. Revista Ecosistemas, 11(2). Recuperado de: https://scholar.google.es/scholar?hl=es\&as_sdt=0\%2C5\&q=que+es+el+desarrollo+sosteni $\underline{\text { ble\&btnG}=}$

Bassotti, G. (2003). Factores de calidad en ecoturismo. Estudios y Perspectivas en Turismo, $12,7-23$.

Bravo, E. (2002). El convenio sobre diversidad biológica diez años después. Grupo Semillas, Documentos, sl. Recuperado de: https://scholar.google.es/scholar?start=10\&q=el+Convenio+de+Bonn\&hl=es\&as_sdt=0,5 
Bringas, N. \& Ojeda, L. (2000). El ecoturismo: ¿una nueva modalidad del turismo de masas? Economía, sociedad y territorio, 2(7).

Constitución de la República Bolivariana de Venezuela (2000). Venezuela. La Piedra.

Constitución de la República del Ecuador. (2008). Constitución del Ecuador. Constitucion del Ecuador.

Corraliza, J. A., Martín, R., Moreno, M., \& Berenguer, J. (2002). El estudio de la conciencia ambiental. Medio ambiente, (40), 36-39.

de Colombia, C. P. (1991). República de Colombia. Ministerio de justicia y del Derecho. Art, 44.

Dialhy, C., \& Herrera, M. (2011). Línea base de conocimiento sobre el estado actual de las tortugas marinas en el Ecuador. Boletín Especial, 2(2).

Eagles, P. F., McCool, S. F., \& Haynes, C. D. (2002). Turismo sostenible en áreas protegidas. Directrices de planificación y gestión. Preparado para el Programa de las Naciones Unidas para el Medio Ambiente, la Organización Mundial del Turismo y la UICN-Unión Mundial para la Naturaleza. Organización Mundial del Turismo. Madrid. Recuperado de: https://www.e-unwto.org/doi/pdf/10.18111/9789284406432

Escobar, A. (1995). El desarrollo sostenible: diálogo de discursos. Ecología política, (9), 725.

Fennell, D. (2001). “A content analysis of ecotourism definitions". Current Issues in Tourism, Vol. 4, pp. 403-421.

Flores, J. C. M. (2008). El turismo en los espacios naturales protegidos españoles, algo más que una moda reciente. Boletín de la Asociación de Geógrafos Españoles, (46), 291-304.

Fonseca, F. A. O., James, J., \& Márquez, G. (2013). Visión comunitaria de los beneficios derivados del ecoturismo en el Parque Nacional Natural Amacayacu (Amazonas, 
Colombia). Gestión y Ambiente, 16(1), 17-32. Recuperado de: https://revistas.unal.edu.co/index.php/gestion/article/view/33837/40481

Gurría, M. (1996). Turismo Moderno de Orientación Ecológica, Un caso de estudio en Colombia. Estudios y Perspectivas en Turismo, Vol. 5, № 4, Buenos Aires: Centro de Investigaciones y Estudios Turísticos. Recuperado de: https://dialnet.unirioja.es/servlet/articulo?codigo=5026289

Ibáñez, R. \& Reyna, C. (2011). Teoría general del turismo: un enfoque global y nacional. México: UABCS y Academia de Investigación Turística. Recuperado de: http://uabcs.mx/difusion2017/files/libros/pdf/184_20160908023838.pdf

Maldonado, C. (2006). Turismo y comunidades indígenas: Impactos, pautas para autoevaluación y códigos de conducta. Geneva: OIT. Recuperado de: http://www.ilo.int/wcmsp5/groups/public/---ed_emp/---emp_ent/--ifp_seed/documents/publication/wcms_117521.pdf

Molina, E. (2012). Estudio comparativo de los sitios Ramsar en el Ecuador como oferta turística del Patrimonio Natural del Ecuador. RICIT: Revista Turismo, Desarrollo y Buen Vivir, (3), 45-81

OMT (1998). Turismo Panorama 2020. Nuevas previsiones de la Organización Mundial del Turismo. Avance actualizado, junio, Madrid, OMT.

Van Dam, C. (2001). La Equidad en el Convenio sobre Diversidad Biológica: transitando un campo minado. Debate Agrario, (33), 91-122. Recuperado de: http://www.cepes.org.pe/debate/debate33/07-articulo-da33.pdf

Vanegas, G. (2006). Ecoturismo instrumento de desarrollo sostenible. Recuperado de http://tesis.udea.edu.co/bitstream/10495/149/1/EcoturismoInstrumentoDesarrolloSostenibl e.pdf 